УДК 621.314

Батыгин Ю.В., д.т.н., Еремина Е.Ф., к.Т.н., Шиндерук С.А, к.т.н., Чаплыгин Е.А., к.т.н. Харьковский национальный автомобильно-дорожный университет

\title{
АНАЛИЗ ЭЛЕКТРОМАГНИТНЫХ ПРОЦЕССОВ В РЕЗОНАНСНОМ УСИЛИТЕЛЕ ЭЛЕКТРИЧЕСКОЙ МОЩНОСТИ
}

Целью настоящей работь было предложение $u$ теоретическое обоснование принциииальной работоспособности схемы трансформатора реактивной электрической мощности, состоящей из двух последовательных резонансных контуров с регулируемым уровнем электромагнитной связи между ними. Получены базовые аналитические выражения для характеристики электромагнитных проиессов в схеме принятого резонансного преобразователя. Показано, что максимально возможный коэффициент усиления электрической реактивной мощности пропориионален добротности вторичного контура трансформатора. Достоверность полученных результатов подтверждается проведенными экспериментальными исследованиями. Их результаты продемонстрировали, что расхождения теоретических оценок и опытных данных довольно незначительны, и их можно объяснить влиянием внешних электромагнитных полей (суперпозиция полей «выходного» соленоида и трансформатора связи). В иелом, полученные результаты иллюстрируют реальные возможности многократного усиления реактивной электрической мощности в предложенной схеме резонансного трансформатора. Дальнейшие работы предполагают разработку $u$ создание преобразователя реактивной электрической энергии в активную.

Ключевые слова: трансформатор, реактивная мощность, последовательный электрический контур, резонанс напряжений, электромагнитная связь, активная нагрузка

Введение, постановка проблемы. Энергию любой природы, извлекаемую при резонансе из внешней среды, можно назвать «резонансной» энергией. Её существование и, соответственно, обоснованность предложенного термина подтверждаются достаточно многочисленными и убедительными примерами [1]. Не останавливаясь на обсуждении конкретных фактов из цитируемой монографии, можно выделить нечто общее во всех известных проявлениях этой энергии. Так, при резонансе и вне его «физические факторы причинности» наблюдаемых явлений остаются неизменными (амплитуды внешней силы, напряжения, тока и др.). Причём их непосредственные возможности не соответствуют резонансному росту амплитуд динамических характеристик и выполнению работы, требующей дополнительных энергетических затрат. Как представляется из феноменологических соображений, эта дополнительная энергия извлекается из окружающего пространства, она существует и, соответственно, может быть названа «резонансной» энергией. Однако следует подчеркнуть, что термин «резонансная» не исключает возможного и справедливого использования других определений энергии из внешней среды, поскольку в современных научных публикациях отсутствует окончательное научное обоснование происхождения энергии такого рода. Постановка задач настоящего исследования инициирована практическим интересом к резонансным явлениям в электротехнике.

Анализ последних исследований и публикаций. Прежде чем перейти непосредственно к обзору литературы, следует осветить два основных фундаментальных утверждения, на которых строится настоящая работа. Первое утверждение состоит в использовании идеи Тесла о резонансной энергии эфира в окружающем пространстве, которая была воплощена практически в «сверх трансформаторе» напряжения, названном его именем [3]. Второе утверждение состоит в использовании отличительной особенности идеализированной схемы с двумя индуктивно-связанными резонансными контурами. Эта идеализация предполагает нулевое активное сопротивление во вторичном контуре, что позволяет получить усиление напряжения и тока, что в конечном итоге означает усиление именно электрической мощности [4].

Более подробно начнем с идей Н. Тесла. Наиболее эффективное предложение конвертера резонансной энергии с коэффициентом преобразования более чем в 1000 раз было запатентовано ещё в начале прошлого века и названо по имени его изобретателя

() Батыгин Ю.В., д.т.н., Еремина Е.Ф., к.т.н., Шиндерук С.А, к.т.н., Чаплыгин Е.А., к.т.н. 
«трансформатором Тесла» [5]. Последний состоит из двух индуктивно связанных резонансных контуров. Физически отсутствие замкнутой цепи с нагрузкой означает, что между выходными клеммами катушки могут возбуждаться только токи смещения с довольно малыми амплитудами. Работает трансформатор Тесла исключительно как «сверх» усилитель напряжения (но не электрической мощности). Его исследованиям посвящено достаточно большое количество публикаций. Так, информация о многочисленных изобретениях, связанных с трансформатором Тесла, содержится в монографиях [3, 5]. Здесь приведены оригинальные не редактированные рисунки, которые касаются переменного тока, его источников, беспроводной передачи электрической энергии, различных конструкций электрических генераторов и многих других очень интересных и практически полезных предложений. Оптимальная конструкция и реальные пути повышения эффективности трансформатора Тесла подробно описаны в работе [6], где детально обсуждаются возможности достижения оптимума за счёт модификации основных компонентов оборудования. Исследования, направленные на повышение амплитуд высоковольтных сигналов на выходе вторичной обмотки трансформатора, на появление высших типов колебаний в спектре возбуждаемого напряжения и др. описаны авторами работы [7]. Принципиально новые физические подходы к решению проблем энергетики без известных её источников обсуждаются авторами работы [8]. Здесь освещены запатентованные в разных странах мира предложения нетрадиционных способов получения, так называемой, «естественно возобновляемой» энергии. В отличие от известных подходов к решению проблем энергетики, предметом дискуссий являются так называемая «радиантная энергия» трансформатора Тесла, «свободная энергия» внешней среды, «холодное электричество», «энергетика без топлива», «энергия эфира» и т.д. Многие предложения авторов цитируемых публикаций были уже апробированы, многие, возможно, будут реализованы в будущем. Интересно отметить, что электродвигатель американского инженера Э. Грея потреблял не более $\sim 5 \%$ энергии источника.

Прежде чем перейти ко второму фундаментальному утверждению об использовании отличительной черты идеализированной схемы с двумя индуктивно-связанными резонансными контурами, следует упомянуть опубликованные работы аналогичной направленности $[9,10]$. Так, патент [9] защищает изобретения резонансного трансформатора и резонансного усилителя. Но как показал строгий анализ запатентованных устройств, их практическая реализация очень сложна и даже сомнительна.

Первая попытка предложения и теоретического анализа возможности создания резонансного трансформатора реактивной электрической мощности представлена в работе [10]. Сущность предложения в цитируемой публикации состоит в следующем. Если трансформатор Тесла дополнить последовательным включением сосредоточенной ёмкости в цепь вторичной обмотки, то полученная схема из двух индуктивно связанных активно-реактивных контуров в режиме «резонанса напряжений» при определённых условиях будет представлять собой уже трансформатор с возможностями усиления не только напряжения, но и тока. Принципиальное доказательство этого утверждения дано в работе [4]. Авторами цитируемого источника показано, что в идеализации, когда активное сопротивление вторичного контура равно нулю, в нём протекает ток конечной величины $\left(I_{2} \neq 0\right)$. А в первичном контуре ток будет отсутствовать $\left(I_{1}=0\right)$. Данную ситуацию авторы интерпретируют следующим образом: при выполнении резонансных условий возбуждаемые токи «устанавливаются такими, чтобы э.д.с. взаимной индукции со стороны второго контура на первый контур уравновесила приложенное напряжение. Физически, по своему характеру, этот случай аналогичен резонансу токов в контуре без потерь. Таким образом, при нулевом токе в первичном контуре и ненулевом во вторичной цепи появляется возможность получения достаточно больших коэффициентов преобразования, но уже для тока, а не только для напряжения.

Заканчивая настоящий обзор, следует отметить, что активно-реактивные электрические контуры широко распространены. Они являются непременными компонентами современных высоковольтных устройств в оборудовании для современных промышленных технологий (например, магнитно-импульсная обработка металлов $[11,12]$ ), а также в экспериментальных комплексах для исследования процессов взаимодействия полей и тонкостенных проводников (например, особенности поведения листовых ферромагнетиков в проникающих электромагнитных полях [13]).

Нерешённая ранее часть общей проблемы. Существенный недостаток рассмотренной схемы двухконтурного резонансного трансформатора (предложенной авторами [4]) состоит в

() Батыгин Ю.В., д.т.н., Еремина Е.Ф., к.т.н., Шиндерук С.А, к.т.н., Чаплыгин Е.А., к.т.н. 
том, что она не может быть реализована на практике. В действительности активное сопротивление вторичной цепи не может быть равным нулю. Тем не менее, рассмотренная идеализация может быть положена в основу уже практической реализации резонансного трансформатора реактивной электрической мощности. Он должен состоять из двух последовательных индуктивно связанных резонансных контуров, но с активным сопротивлением конечного значения. Уровень электромагнитной связи между ними должен регулироваться так, чтобы избежать передачи резонансной энергии из вторичной цепи в первичную. Согласно рекомендациям, Н. Тесла, эта связь должна быть достаточно слабой. Это означает, что возбуждение вторичной цепи должно осуществляться небольшими «порциями» энергии и предотвращать ее возврат в первичный контур. «Вывод» генерируемой энергии может быть реализован из индуктивности вторичной цепи или из её емкости.

Формулировка цели статьи. Целью настоящей работы является теоретическое обоснование принципиальной работоспособности предложенной ранее [14] схемы трансформатора реактивной электрической мощности, состоящей из двух последовательных активно-реактивных резонансных контуров с регулируемым уровнем электромагнитной связи между ними. Научная новизна определяется сформулированной целью, достижение которой основано на выводах обобщённого теоретического анализа и экспериментах, выполненных для действующей модели предлагаемого трансформатора реактивной электрической мощности.

Методика проведения исследований. Проведенные исследования включают две составляющие. Первая - это вывод аналитических зависимостей для описания протекающих электромагнитных процессов и численные оценки их характеристик для создания действующей модели трансформатора реактивной электрической мощности. Вторая составляющая предполагает экспериментальные исследования разработанной модели трансформатора, что необходимо для проверки достоверности теоретических результатов и формулировки рекомендаций при проектировании реальных резонансных преобразователей реактивной электрической мощности.

Особо следует отметить, что аналогично работам $[4,13]$ все нижеприведенные расчетные соотношения базируются на физически «прозрачных» положениях и строгом математическом подходе с использованием аппарата теории цепей. И поскольку практический интерес представляет работа исследуемого объекта в стационарном режиме с гармоническими токами и напряжениями, в работе применялся исключительно метод комплексных амплитуд без какихлибо физических гипотез о строении окружающего мира и физических причинах резонансных явлений $[4,13]$.

Получение научных результатов.

Постановка задач расчёта и принимаемые допущения:

- На рис. 1 представлена схема замещения усилителя из двух последовательных резонансных контуров с трансформатором связи между ними. Индуктивности первичной и вторичной обмоток - $L_{1 \mathrm{~T}, 2 \mathrm{~T}}$, соответственно.

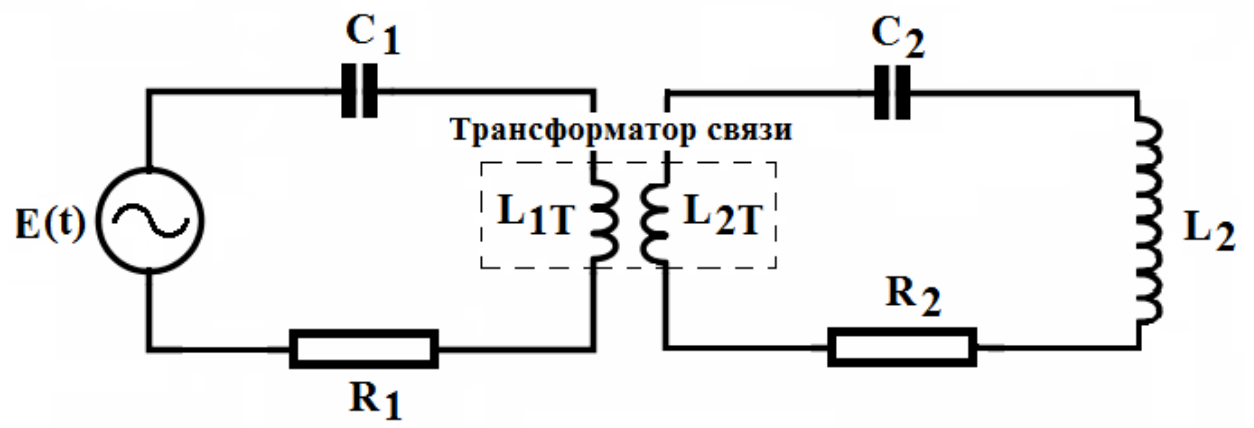

Рис. 1. Принципиальная схема трансформатора реактивной электрической мощности

- Первый «входной» контур: $E(t)=E_{m} \cdot \sin (\omega t)$ - источник гармонического напряжения $\left(E_{m}\right.$ - амплитуда, $\omega$ - частота, $t$ - время) $L_{1 \mathrm{~T}}$ - индуктивность первичной обмотки трансформатора связи между контурами. $C_{1}-$ емкость, активное сопротивление $-R_{1}$.

- Второй «выходной» контур: $L_{2 \mathrm{~T}}-$ индуктивность вторичной обмотки трансформатора связи между контурами, $L_{2}$ - «выходная» индуктивность, $C_{2}$ - емкость, $R_{2}$ - активное сопротивление.

() Батыгин Ю.В., д.т.н., Еремина Е.Ф., к.т.н., Шиндерук С.А, к.т.н., Чаплыгин Е.А., к.т.н. 
Поскольку напряжения на реактивных элементах одинаковы, емкость $-C_{2}$, также может быть «выходным» элементом.

- Частоты первичного и вторичного контуров равны друг другу, так что $\omega_{1}=\omega_{2}=\omega-$ резонансная частота системы.

Вывод расчетных соотношений.

Система уравнений, описывающая процессы в контурах схемы замещения предложенного трансформатора, имеет вид $[4,13]$ :

$$
\left\{\begin{array}{l}
E=I_{1} \cdot\left(i\left(\omega L_{1 T}-\frac{1}{\omega C_{1}}\right)+R_{1}\right)+i \omega M_{12} \cdot I_{2} ; \\
-i \omega M_{12} \cdot I_{1}=\left(i\left[\omega \cdot\left(L_{2 T}+L_{2}\right)-\frac{1}{\omega C_{2}}\right]+R_{2}\right) \cdot I_{2} ;
\end{array}\right.
$$

где $I_{1,2}-$ токи в контурах, $I_{2}=-i \cdot \frac{E}{\left(\omega M_{12}\right)}-$ взаимная индуктивность обмоток трансформатора связи между контурами, $k \in[0,1]-$ коэффициент электромагнитной связи.

При выполнении резонансных условий $\left\{\left(\omega \cdot\left(L_{2 T}+L_{2}\right)-\frac{1}{\omega C_{2}}\right)=0,\left(\omega L_{1 T}-\frac{1}{\omega C_{1}}\right)=0\right\}$ система уравнений (1) принимает вид:

$$
\left\{\begin{array}{l}
E=I_{1} \cdot R_{1}+i \omega M_{12} \cdot I_{2} \\
-i \omega M_{12} \cdot I_{1}=R_{2} \cdot I_{2}
\end{array}\right.
$$

Выражение для возбуждаемых токов получим из (2).

$$
\left\{\begin{array}{l}
I_{1}=E \cdot\left(\frac{R_{2}}{\left.\left(\omega M_{12}\right)^{2}+R_{1} \cdot R_{2}\right)}\right. \\
I_{2}=-i \cdot \frac{E}{Z}
\end{array}\right.
$$

где $Z=\frac{\left(\omega M_{12}\right)^{2}+R_{1} \cdot R_{2}}{\omega M_{12}}$

Следует отметить, что $Z$ имеет смысл модуля эквивалентного индуктивного сопротивления, которое связывает напряжение источника питания с резонансным током во вторичном контуре. Как следует из выражения для $I_{2}$ из (4) между ними имеет место строгая индуктивная связь.

Переход к пределу $R_{2} \rightarrow 0$ показывает, что $I_{1}=0$ и $I_{2}=-i \cdot \frac{E}{\left(\omega M_{12}\right)}$. Это полностью согласуется с результатом идеализации в работе [4].

Для проверки достоверности полученных зависимостей можно найти соотношение напряжения на индуктивности «выходного» контура и напряжения на индуктивности первичного контура. Это необходимо для сравнения с аналогичным соотношением в трансформаторе Тесла [5].

Согласно определению и формулам (3), мы можем найти, что

$$
k=\left|\frac{U_{L_{2}}=\left(\omega L_{2} \cdot I_{2}\right)}{U_{L_{1 T}}=\left(\omega L_{1 T} \cdot I_{1}\right)}\right|=k_{m p} \cdot Q_{2},
$$

где $U_{L_{1 T, 2}}-$ напряжения на индуктивности $L_{1 \mathrm{~T}, 2}, \quad$ соответственно, $k_{m p}=k_{12} \cdot \sqrt{\frac{L_{2 T}}{L_{1 T}}}-$ коэффициент преобразования электромагнитной индукции, $Q_{2}=\frac{\omega L_{2}}{R_{2}}-$ добротность вторичного контура (без индуктивности первичной обмотки трансформатора связи), определяющая количественный вклад резонансных явлений в процессе преобразования напряжения.

Следует отметить, что результат (4) полностью согласуется с качественной оценкой аналогичного параметра для трансформатора Тесла (прототип настоящего авторского предложения) в статьях [5]. Данный параметр был определен изобретателем в результате 
обобщения многочисленных экспериментальных данных. Он утверждал, что эффективность его трансформатора определяется отношением индуктивного сопротивления вторичного контура к его активному сопротивлению.

Прежде чем перейти к количественной интерпретации полученных характеристик, вернемся ко второму соотношению из (3) для тока во вторичном контуре $-I_{2}$.

Очевидно, что функциональная зависимость $Z=Z\left(\omega M_{12}\right)$ должна иметь минимум, который будет определять максимум тока $I_{2}$ относительно аргумента $\left(\omega M_{12}\right)$. [16]:

Известное необходимое условие существования экстремума для $Z=Z\left(\omega M_{12}\right)$ имеет вид

$$
\frac{d Z\left(\omega M_{12}\right)}{d\left(\omega M_{12}\right)}=\frac{\left(\omega M_{12}\right)^{2}-R_{1} \cdot R_{2}}{\left(\omega M_{12}\right)^{2}}=0 .
$$

Как следует из выражения (5), модуль эквивалентного сопротивления $Z=Z\left(\omega M_{12}\right)$, как функция аргумента $\left(\omega M_{12}\right)$, достигает минимума, если $\left(\omega M_{12}\right)_{\min }=\sqrt{R_{1} \cdot R_{2}}$ и минимум сопротивления будет равен $Z_{\text {min }}=2 \sqrt{R_{1} \cdot R_{2}}$.

Для схемы предложенного резонансного трансформатора условие реализации минимального сопротивления $\mathrm{Z}_{\min }$ имеет вид:

$$
\omega \cdot k_{12} \cdot \sqrt{L_{1 T} \cdot L_{2 T}}=\sqrt{R_{1} \cdot R_{2}}
$$

Значение коэффициента электромагнитной связи, обеспечивающего максимальный ток вторичной обмотки, можно получить из выражения (6).

$$
k_{12-\max }=\sqrt{\frac{R_{1} \cdot R_{2}}{\left(\omega \cdot L_{1 T}\right) \cdot\left(\omega \cdot L_{2 T}\right)}} .
$$

Физически, минимум эквивалентного сопротивления и соответствующий максимуму эффективности резонансного усилителя можно объяснить минимально возможной перекачкой энергии из вторичного контура в первичный. Причём данное положение вещей достигается соответствующим уровнем электромагнитной связи между контурами согласно формуле (7).

Следует особо отметить, что полученное выражение для коэффициента уровня электромагнитной связи позволяет выполнить предварительные оценки, необходимые для практической реализации рекомендаций Н. Тесла, согласно которым резонансное возбуждение «выходного» контура должно происходить путем достаточно небольших электрических воздействий со стороны «входного» контура с рабочей частотой, равной собственным частотам контуров усилителя.

С учетом полученных соотношений все необходимые зависимости в режиме максимума тока и максимума мощности в «выходном» элементе предложенного резонансного трансформатора реактивной электрической можно объединить следующим образом.

$$
\left\{\begin{array}{l}
I_{1 \mathrm{~m}}=\frac{E}{2 R_{1}} ; \\
U_{L_{1 T} \mathrm{~m}}=E \cdot \frac{Q_{1}}{2} ; \\
P_{1 \mathrm{~m}}=\frac{E^{2}}{2 R_{1}} ; \\
I_{2 \mathrm{~m}}=\frac{E}{2 \cdot \sqrt{R_{2} \cdot R_{1}}} ; \\
U_{L_{2} \mathrm{~m}}=E \cdot \frac{\left(\omega \cdot L_{2}\right)}{2 \cdot \sqrt{R_{2} \cdot R_{1}}} \\
P_{2 \mathrm{~m}}=\frac{E}{R_{1}} \cdot \frac{Q_{2}}{4}
\end{array}\right.
$$

где $Q_{1}=\frac{\left(\omega \cdot L_{1 T}\right)}{R_{1}}-$ добротность первичного контура.

Теперь, модуль отношения токов в контурах:

() Батыгин Ю.В., д.Т.н., Еремина Е.Ф., к.т.н., Шиндерук С.А, к.т.н., Чаплыгин Е.А., к.т.н. 


$$
\left|\frac{I_{2}}{I_{1}}\right|=\sqrt{\frac{R_{1}}{R_{2}}}
$$

Из (9) следует, что отношение токов обратно пропорционально корню квадратному из отношения активных сопротивлений в контурах, т.е. соотношение между возбуждаемыми токами определяется уровнем рассеяния энергии.

Отношение выходной и входной мощности определяет максимальный коэффициент преобразования мощности в предлагаемом резонансном усилителе:

$$
K_{\text {max }}=\frac{P_{2}}{P_{1}}=\frac{Q_{2}}{2}
$$

Отметим, что, как следует из (10), максимальный коэффициент усиления достигается за счет резонансного возбуждения предложенного трансформатора. Некоторые результаты анализа зависимостей (8-10), описывающих электромагнитные процессы в режиме максимума возможной эффективности преобразования электрической энергии, представлены в нижеследующих обобщающих положениях.

- Полученные результаты представляют собой количественные показатели действенности резонансного трансформатора реактивной электрической мощности из двух индуктивно связанных последовательных $R L C$ - контуров.

- В отличие от трансформатора Тесла в принятой схеме преобразователя имеет место трансформация уже не только напряжения, а мощности гармонических токов и напряжений.

- Связь между резонансным током и напряжением трансформатора реактивной электрической мощности имеет индуктивный характер и эквивалентное сопротивление связи можно интерпретировать как эквивалентную индуктивность всей системы в целом.

- Соотношение между токами, возбуждаемыми в отдельных контурах схемы предложенного трансформатора, определяется уровнем рассеяния электромагнитной энергии.

- При соответствующем выборе параметров принятая схема резонансного преобразователя может рассматриваться как усилитель электрической мощности с максимально возможным коэффициентом усиления $-K_{\max }=0.5 \cdot\left(\frac{\omega \cdot L_{2}}{R_{2}}\right), \omega, L_{2}, R_{2}-$ резонансная частота, выходная индуктивность, активное сопротивление вторичного контура.

- Физически, максимум усиления мощности резонансным преобразователем обусловлен минимально возможной перекачкой энергии из вторичного выходного контура в первичный контур с источником входного гармонического напряжения.

Численные оценки и экспериментальные результаты. Изложение последующего материала будет построено на сопоставлении численных оценок и полученных экспериментальных результатов.

Начнём с описания элементной базы экспериментальной модели, принципиальная эквивалентная схема которой приведена на рис. 1.

- Первый «входной» контур: $E(t)=E \cdot \sin (\omega t)$ - источник гармонического напряжения с амплитудой $-E=1 \mathrm{~B}$ и рабочей частотой $-\omega=2 \pi \cdot 25$ кГц, $R_{1}=0.1$ Ом - активное сопротивление, $C_{1}=2.763$ мкФ - ёмкость, $L_{1 \mathrm{~T}}=14.8$ мкГн - индуктивность первичной обмотки трансформатора связи между контурами.

- Блок трансформатора связи.

- Второй «выходной» контур: $L_{2 \mathrm{~T}}=14.8$ мкГн - индуктивность вторичной обмотки трансформатора связи между контурами, $C_{2}=0.22$ мкФ - ёмкость, $L_{2}=169.2$ мкГн «выходная» индуктивность, $R_{2}=0.35$ Ом - активное сопротивление.

Вычисления и измерения.

Первый контур.

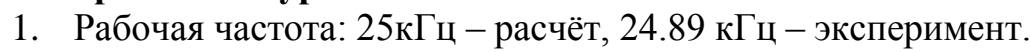

2. Коэффициент электромагнитной связи между обмотками: $\mathrm{k}_{12 \text {-max }} \approx 0.08$ - расчёт, $\mathrm{k}_{12-}$ $\max \approx 0.093$ - эксперимент.

3. Возбуждаемый ток: $I_{1 \mathrm{~m}}=5.0 \mathrm{~A}$ - расчёт, $I_{1 \mathrm{~m}}=5.46 \mathrm{~A}$ - эксперимент. 
4. Напряжение на первичной обмотке трансформатора связи между обмотками $U_{L_{1 T m}}=11.0 \mathrm{~B}$ - расчёт, $U_{L_{1 T m}}=11.58 \mathrm{~B}$ - эксперимент.

5. Мощность от источника $-\mathrm{P}_{1 \mathrm{~m}}=5.0 \mathrm{BT}-$ расчёт, $\mathrm{P}_{1 \mathrm{~m}}=5.46 \mathrm{BT}-$ эксперимент.

Второй контур.

6. Рабочая частота: 25 кГц - расчёт, 24.93 кГц - эксперимент.

7. Возбуждаемый ток: $I_{2 \mathrm{~m}}=2.7 \mathrm{~A}$ - расчёт, $I_{2 \mathrm{~m}}=3.39 \mathrm{~A}$ - эксперимент.

8. Напряжение на первичной обмотке трансформатора связи между обмотками: $U_{L 2 T m}=$ $6.19 \mathrm{~B}$ - расчёт, $U_{L_{2} T m}=5.8 \mathrm{~B}$ - эксперимент.

9. «Выходная» мощность на индуктивности $-L_{2}: P_{2 \mathrm{~m}}=189.2 \mathrm{BA}-$ расчёт, $P_{2 \mathrm{~m}}=191.2 \mathrm{BA}$.

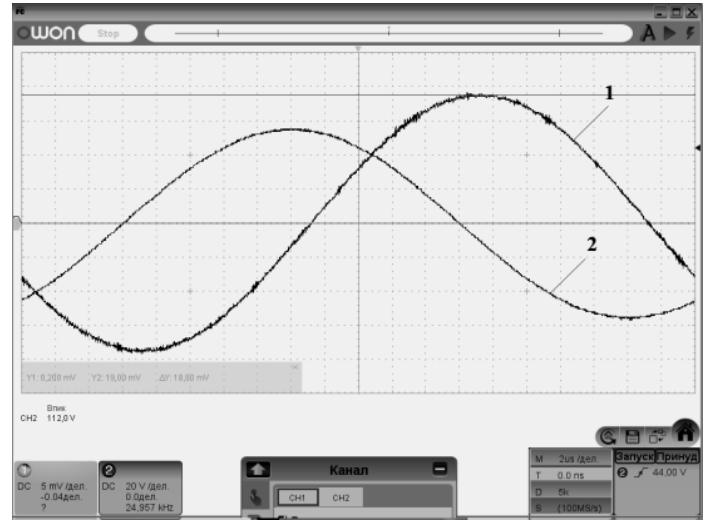

Рис. 3. Осциллограмма: ток в «выходной» индуктивности - 1, напряжение - 2

\section{Интегральные показатели.}

1. Отношение «выходной» мощности преобразователя к «выходной» мощности первого контура $\frac{U_{L 2 \mathrm{~m}} \cdot J_{2 \mathrm{~m}}}{U_{L 1 \mathrm{~m}} \cdot J_{1 \mathrm{~m}}}$ составляет 3,2 по результатам эксперимента и 3,3 из расчета (расхождение $\sim 3 \%$ ).

2. Отношение «выходной» мощности преобразователя к «выходной» мощности источника - коэффициент усиления реактивной электрической мощности $K=\frac{U_{L 2 \mathrm{~m}} \cdot J_{2 \mathrm{~m}}}{E \cdot J_{1 \mathrm{~m}}}$ составляет

35 по результатам эксперимента и 37,8 из расчета, (расхождение $~ 9 \%$ ).

\section{Выводы и перспективы дальнейшего развития в исследованном направлении.}

1. Теоретически и экспериментально обоснована принципиальная работоспособность принятой схемы трансформатора реактивной электрической мощности, состоящего из двух последовательных резонансных контуров с регулируемым уровнем электромагнитной связи между ними.

2. Получены базовые аналитические выражения для анализа электромагнитных процессов в схеме принятого резонансного преобразователя.

3. Показано, что максимально возможный коэффициент усиления электрической реактивной мощности равен $-K_{\max }=\frac{Q_{2}}{2}$, где $Q_{2}=\frac{\omega \cdot L_{2}}{R_{2}}$ добротность, $\omega, L_{2}, R_{2}-$ резонансная частота, «выходная» индуктивность, активное сопротивление «выходного» контура предлагаемого резонансного трансформатора реактивной электрической мощности.

4. Отклонения результатов измерений в сравнении вычислениями довольно малы и их можно объяснить влиянием внешних электромагнитных полей (суперпозиция полей «выходного» соленоида и трансформатора связи).

5. Проведенные теоретические и экспериментальные исследования согласуются с рекомендациями Н. Тесла, согласно которым резонансная «накачка» вторичного «выходного» контура должна производиться достаточно малыми электрическими воздействиями со стороны первичного «входного» контура на частоте, равной собственным частотам контуров усилителя. 
6. В целом, полученные результаты иллюстрируют реальные возможности многократного усиления реактивной электрической мощности (в $\sim 33 \div 35$ раз) в предложенной схеме резонансного трансформатора.

7. Дальнейшие работы, в конечном итоге, предполагают создание действующей модели преобразователя реактивной электрической энергии в активную, который при подключении к выходному элементу трансформатора реактивной мощности позволит создать двухступенчатый резонансный усилитель активной электрической мощности без каких-либо дополнительных источников энергии.

\section{Информационные источники}

1. Whittaker, E.T. A History of the Theories of Aether \& Electricity: Two Volumes Bound As One Paperback - May 17, 2017. Manufactured in the US by LSC Communications. 2017.

2. Beneson, W., Haris, J.W., Stocer, H. \& Lutz H. (2019). Handbook of Physics. Switzerland: Springer Nature AG. 2019

3. Gunderman R.. Tesla, the Man, Inventor and the Father of Electricity. Publisher: Carton Books Ltd. 2016.

4. Демирчян К.С. Теоретические основы электротехники. Том 1./ К.С.Демирчян, Н.В.Коровкин, Л.Р.Нейман, Чечурин В.Л. - Санкт-Петербург: Издательство «Питер», 2009. $463 \mathrm{c}$. 2016.

5. Tesla N. My Inventions, and Other Writing. Dover Drift Edition. Dover Publication Inc.

6. Voitkans J., Voitkans A. Tesla Coil Theoretical Model and its Experimental Verification, Published Online: 2015-03-5.

7. Craven R.M., Smith I.R., and Novac B.M., Optimizing the secondary coil of a Tesla transformer to improve spectral purity. IEEE Transactions on Plasma Science. 2014. 42(1), pp. 143148 .

8. Batygin Yu.V., Shinderuk S.A., Serikov G.S., Karabuta V.O., Modern Power Engineering in the Light of the New Physical Representations and Technical Realizations. - A brief review.// Міжнародний журнал «Світлотехніка та електроенергетика» СТЕЕ 2019. - №1 (54), - pp. 40-46.

9. Лиховид Ю.М. (2015). Патент України 103215. Київ: Державна служба інтелектуальної власності України.

10. Stepanov A.A, The resonant transformer. Patent RU 2418333 C1, 10.05.2011

11. Electromagnetic Forming. (C) Fraunhofer Institute for Mashine Tools and Forming Technology IWU 2016.

12. D. Gayakward, M.K. Dargar, P.K. Sharma and others. A Review on Electromagnetic Forming Processes. Procedia Materials Science (ICMPC 2014) pp. 520-527.

13. Батигін, Ю.В., Гнатов, А.В. Особливості збудження електромагнітних сил при магнітно-імпульсній обробці листових феромагнетиків //Технічна електродинаміка, 2012. №1. - C. 71-77.

14. Батыгин Ю.В., Шиндерук С.А., Сериков Г.С., Ерёмина Е.Ф. Резонансный усилитель электрической мощности. Эксперименальные исследования/ «Перспективні технології та прилади». Збірник наукових праць. - Луцьк: ЛНТУ, 2018. - Випуск №13 - С. 18-24.

15. Thomson, J.J., Elements of the Mathematical Theory of Electricity and Magnetism. Publisher: Wentworth. 2016.

16. Kantorovich L. Mathematics for Natural Scientists. Fundamentals and Basics. Springer Nature Switzerland AG. 2018. - 526 P.

Батигін Ю.В., д.т.н., Єрьоміна О.Ф, к.т.н., Шиндерук С.О, к.т.н., Чаплигін Є. О., к.т.н. Харківський національний автомобільно-дорожній університет

\section{АНАЛІЗ ЕЛЕКТРОМАГНІТНИХ ПРОЦЕСІВ В РЕЗОНАНСНИХ ПІДСИЛЮВАЧАХ ЕЛЕКТРИЧНОЇ ПОТУЖНОСТІ}

Метою иієї роботи була пропозиція $i$ теоретичне обтрунтування принципової працездатності схеми трансформатора реактивної електричної потужності, щчо складається $з$ двох послідовних резонансних контурів 3 регульованим рівнем електромагнітного зв'язку між ними. Отримано базові аналітичні вирази для характеристики

(С) Батыгин Ю.В., д.т.н., Еремина Е.Ф., к.т.н., Шиндерук С.А, к.т.н., Чаплыгин Е.А., к.т.н. 
електромагнітних процесів в схемі прийнятого резонансного перетворювача. Показано, що максимально можливий коефіцієнт посилення електричної реактивної потужності пропориійний добротності вторинного контуру трансформатора. Достовірність отриманих результатів підтверджується проведеними експериментальними дослідженнями. Ïх результати продемонстрували, щь розбіжності теоретичних оцінок і дослідних даних досить незначні, $і$ їх можна пояснити впливом зовнішніх електромагнітних полів (суперпозиція полів «ви-перехідного» соленоїда і трансформатора зв'язку). В иілому, отримані результати ілюструють реальні можливості багаторазового посилення реактивної електричної потужності в запропонованій схемі резонансного трансформатора. Подальші роботи припускають розробку і створення перетворювача реактивної електричної енергії в активну.

Ключові слова: трансформатор, реактивна потужність, послідовний електричний контур, резонанс напруг, електромагнітна зв'язок, активне навантаження

Yu.V. Batygin, Sc.D., E.F. Yeryomina, Ph.D., S.A. Shynderuk, Ph.D., E.A. Chaplygin, Ph.D. Kharkov national automobile-highway university

\section{ANALYSIS OF ELECTROMAGNETIC PROCESSES IN A RESONANT ELECTRIC POWER AMPLIFIER}

The purpose of this work was to propose and theoretically substantiate the fundamental operability of the reactive power transformer circuit, consisting of two series resonant circuits with an adjustable level of electromagnetic coupling between them. Basic analytical expressions for the characteristics of electromagnetic processes in the circuit of the adopted resonant converter are obtained. It is shown that the maximum possible amplification factor of electric reactive power is proportional to the quality factor of the secondary circuit of the transformer. The reliability of the results obtained is confirmed by the conducted experimental studies. Their results showed that the discrepancies between theoretical estimates and experimental data are rather insignificant, and they can be explained by the influence of external electromagnetic fields (superposition of the fields of the "output" solenoid and the coupling transformer). In general, the results obtained illustrate the real possibilities of multiple amplification of reactive electrical power in the proposed resonant transformer circuit. Further work involves the development and creation of a converter of reactive electrical energy into active energy.

Key words: transformer, reactive power, series electric circuit, voltage resonance, electromagnetic coupling, active load 